\title{
Article
}

\section{Task-response times, facilitating and inhibiting factors in cross-signing}

\author{
Zeshan, Ulrike
}

Available at http://clok.uclan.ac.uk/26398/

Zeshan, Ulrike ORCID: 0000-0002-8438-3701 (2019) Task-response times, facilitating and inhibiting factors in cross-signing. Applied Linguistics Review, 10 (1). pp. 9-29. ISSN 1868-6303

It is advisable to refer to the publisher's version if you intend to cite from the work. http://dx.doi.org/10.1515/applirev-2017-0087

For more information about UCLan's research in this area go to http://www.uclan.ac.uk/researchgroups/ and search for <name of research Group>.

For information about Research generally at UCLan please go to http://www.uclan.ac.uk/research/

All outputs in CLoK are protected by Intellectual Property Rights law, including Copyright law. Copyright, IPR and Moral Rights for the works on this site are retained by the individual authors and/or other copyright owners. Terms and conditions for use of this material are defined in the policies page. 
Task-response times, facilitating and inhibiting factors in cross-signing

\author{
Abstract
}

This paper reports on data from the "cross-signing" strand of a research project on Sign

Multilingualism. Cross-signing investigates the ad-hoc improvised conversations of small groups of deaf sign language users who do not have fluency in any shared language. Participants were filmed in pairs when they met for the very first time, and after a contact period of 4-6 weeks together as a group.

The deaf signers involved in this study are from the UK, Jordan, Indonesia, Japan, India, and Nepal. All signers are highly fluent in their own sign language, with varying competence in a language of literacy from their home country, but minimal or no overlapping competence in International Sign, English, or any other shared language between them. The participants used a wide range of multilingual and multimodal communicative resources, including their own and invented signs, fingerspelling, pointing, mouthing, gesture/mime, and various representations of writing (Zeshan 2015).

The article considers quantitative data from signed interactions during a picture-based elicitation game. While the overall response times taken by participants for completing the elicitation game are reduced at the end of the contact period compared to the initial contact, differentiating factors are at work that lead to different degrees of response time reduction in the individual signers. As a step towards explaining these patterns, the article explores insights into factors that may inhibit or facilitate communication between cross-signers, such as extent of contact between signers, typological distance between sign languages, or the use of literacy. Moreover, the data suggest a cumulative impact of these factors.

\title{
1. Introduction
}

Cross-signing was first defined in 2013 as "communication between sign language users with divergent linguistic backgrounds who have no language in common and minimal experience of international contact" (Zeshan, Sagara and Bradford 2013). That is, although cross-signers may share bits and pieces of signed or spoken/written languages, as well as some communicative strategies and resources, they are not fluent in any shared language. Research on this phenomenon was carried out in 2012 in the UK and in 2014 in India as part of a larger international project on Sign Multilingualism, led by the author as Principal Investigator between 2011 and 2016.

Cross-signing is part of a continuum of contact phenomena between users of different sign languages. At one end of the continuum, we find semi-conventionalised contact varieties in settings where deaf people from different language backgrounds come together regularly, such as international meetings (e.g. World Federation of the 
Deaf, European Union of the Deaf), sports events (e.g. Deaflympics), and conferences (e.g. SIGN, Deaf Academics). This transnational means of communication has developed over time, and has been documented in the sign language literature under the label International Sign (IS) (e.g. McKee and Napier 2002, Rosenstock 2008, Rosenstock and Napier 2015). The linguistic status of International Sign is explicitly debated in recent literature. Hansen (2015) finds that most of the design features of language as originally proposed by Hocket (1960) are valid for communication via International Sign. Mesch (2010:5) reports divergent views about the status of IS from a survey and notes "a distinction between IS within a group who meets regularly and IS as cross-sign communication (ad hoc signing)".

Cross-signing as used in this article refers to this latter situation of ad hoc signing and thus is situated at the opposite end of the continuum with respect to its level of conventionalisation. Cross-signing research investigates the very first stages of transnational contact, and therefore, communication is largely characterised by emergent improvisations rather than relying on any historically evolved shared conventions, as is the case in IS. In Crasborn and Hiddinga (2015), the same distinction is expressed by using the term "international sign" (without capitalisation) as opposed to the more conventionalised International Sign (with capital letters), and the authors note that "this is not a binary distinction but rather a continuum" (p.60). However, these distinctions are not always made in the sign language literature on transnational signing varieties.

As there is an obvious link between the various phenomena on the continuum, Zeshan (2015:254) argues that cross-signing can be seen as "a window into the past of the development of IS" (i.e. the more conventionalised forms of International Sign).

Cross-signing also occupies a peculiar place in the typology of bi- and multilingual communication involving sign languages. Sign bi- and multilingualism can be unimodal, as in the case of signers code-switching between different sign languages that they are familiar with as a group (Zeshan and Panda 2015), or bimodal by virtue of co-using a sign language and a spoken/written language. Crosssigning is multilingual in the sense that signers share the resources from each other's sign languages, as well as parts of any other sign languages they may be familiar with. In addition, cross-signing is explicitly multimodal because signers exploit not only sign languages but also a wider range of other semiotic means, 
including gestures, mime, pointing, ad hoc invented signs, and various representations of writing (Zeshan 2015).

From a wider perspective, work on cross-signing enables us to explore new aspects of how multilingualism manifests itself in signed communication. So far, most research on International Sign has focused on those varieties that have a higher degree of conventionalisation. Moreover, compared to direct face-to-face interactions between signers, there has been much more work on the use of "expository IS", i.e. unidirectional signing for addressing an audience such as in the context of lectures, presentations, and video recordings for online dissemination (see Rosenstock 2015), including work on IS interpreting (e.g. several chapters in Rosenstock and Napier 2015). Cross-signing research, on the other hand, documents the emergent communication between signers in immediate face-to-face contexts. Therefore, this research allows an insight into live, online negotiations taking place during conversations, which are not easily documented in expository IS or in IS interpreting.

Previous research on cross-signing has documented some of the tools and strategies that signers use to constitute an ad hoc shared basis for communication (Zeshan, Sagara and Bradford 2013, Zeshan 2015, Byun et al, forthcoming). In order to add to the emerging picture, this article looks behind the scenes of the observable communicative behaviours, by discussing a number of factors that may be expected to affect participants in cross-signing situations. These factors are considered in conjunction with quantitative response time data derived from an elicitation game, which revealed individual differences between participants. The aim of this article is to consider the possible factors that may be responsible for such differences, and see if any correlations can be identified between the data and the background information that we have gathered about the participants.

\section{Participants and data}

This study was carried out using a set-up where the emerging communication between international deaf signers without fluency in a shared language could be observed over a substantial period of time. Participants were invited to spend time with the research team specifically for the purpose of conducting the research, although the research team also organised a wide range of other activities with the international participants concurrently with the research. 
Research was carried out in two groups with four participants each. Each group included one local participant and three external participants. Group 1 was hosted at the International Institute for Sign Languages and Deaf Studies in Preston, UK, for six weeks, with external participants from Japan, Indonesia, and Jordan. Group 2 stayed for four weeks at the Dr Shakuntala Misra National Rehabilitation University in Lucknow, India, with external participants from Indonesia, Jordan, and Nepal (the Indonesian and Jordanian participants were different individuals in the two groups). All participants were in their 20 s and 30 s, and included six males and two females.

Participants are listed in Table 1. With the exception of local participants from the UK and India, all participants were selected on the basis of having no or minimal competence in written English and IS, thereby ensuring that overlap in communicative repertoires would be minimised. Some of the external participants did have some experience of travelling abroad and/or meeting deaf foreigners in their home country; however, this experience was rather limited, and none of them could be said to be fluent in the more conventionalised forms of IS. All participants were literate to different degrees in the written languages of their home countries.

\begin{tabular}{|l|l|l|l|l|l|}
\hline Group & Alias name & Country & Gender & Native sign language & In-country written language \\
\hline $1(\mathrm{UK})$ & BRT & UK & $\mathrm{F}$ & British SL & English \\
\hline $1(\mathrm{UK})$ & JP & Japan & $\mathrm{M}$ & Japanese SL & Japanese \\
\hline $1(\mathrm{UK})$ & INDO1 & Indonesia & $\mathrm{M}$ & Indonesian SL & Bahasa Indonesia \\
\hline $1(\mathrm{UK})$ & JORD1 & Jordan & $\mathrm{M}$ & Jordanian SL & Arabic \\
\hline 2 (India) & IND & India & $\mathrm{M}$ & Indian SL & Hindi \& English \\
\hline 2 (India) & $\mathrm{NEP}$ & Nepal & $\mathrm{F}$ & Nepali SL & Nepali \\
\hline 2 (India) & INDO2 & Indonesia & $\mathrm{M}$ & Indonesian SL & Bahasa Indonesia \\
\hline 2 (India) & JORD2 & Jordan & $\mathrm{M}$ & Jordanian SL & Arabic \\
\hline
\end{tabular}

Table 1. Cross-signing participants

The project team took many measures to ensure that external participants would have a positive experience of the research. In Group 1, based in the UK, each of the international participants had a local deaf facilitator, a member of the host institute, who was fluent in the sign language of the participants' home countries and known to them before the research began. In Group 2, the Jordanian and Indonesian participants from Group 1 acted as facilitators for new participants from these two 
countries, and the participant from Nepal arrived accompanied by a Nepali facilitator who was fluent in IS and familiar with the location in India. The participants benefited from detailed briefings during the recruitment and research process by their conational deaf facilitators, an extensive parallel programme of skills development, and continuously available informal interpreting and guidance via their facilitators.

At the same time, the research team also recognised the intrinsic value, opportunities and enjoyment that participants would be able to gain from transnational contact with other deaf people. Work by authors such as Green (2015), Kusters and Friedner (2015), and Murray (2008) has pointed to the importance of a transnational deaf collectivity and the unique role that signed communication plays in these settings. For young deaf people from the Global South without any international experience, participating in such a transnational deaf environment over several weeks is a unique opportunity.

During their time in the UK and in India, a considerable range of data collection activities took place (Zeshan and Webster, forthcoming). For the purpose of this article, the relevant data come from a linguistic elicitation game that was conducted twice with all signers within the two groups, once immediately upon their first meeting, and the second time between three to five weeks into the research. The game was in the form of a "director-matcher" task: one participant (the director) describes a picture to the other (the matcher), who then has to identify the target picture from among two or three options of similar pictures. Participants cannot see each other's pictures, and take turns being director and matcher. Figure 1 shows an example of the stimulus materials. 


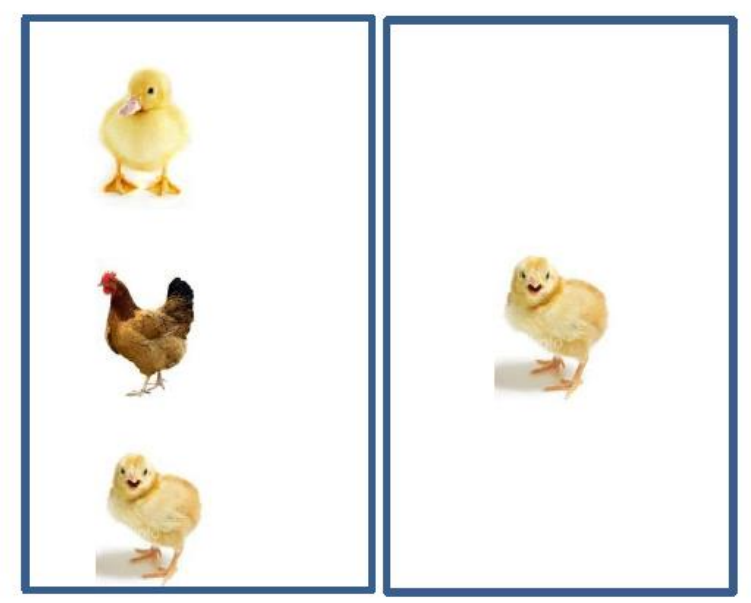

Picture for receiver Picture for signer

Figure 1. Example of stimulus materials

As soon as possible after the first round of data collection, which included ca. 45 minutes of free conversation immediately followed by the first round of the elicitation game, participants undertook introspective interviews conducted by a member of the research team. For this purpose, each participant separately viewed the video recording of the free conversation and discussed it with the researcher. For the international incoming participants, their facilitators acted as interpreters during the session. Participants were asked to comment on the video in terms of what they were trying to convey and how, why they were choosing expressions they used, and what they understood from their interlocutors' utterances. Because of constraints on researcher time and logistics, the interviews were not filmed. Instead, the interviewers produced a summary transcript in English during the session (written in third person) about what the participants were saying. The aim of this methodology was to uncover underlying metalinguistic reasoning and other complexities, and the approach is similar to "stimulated recall interviews" as described in Gass and Mackey (2000) for L2 research. Interestingly, this was also the only way to discover instances where participants had misunderstood each other despite thinking that they had communicated successfully. In this article, the introspective interview data are not analysed, but comments made by participants during the interviews are used in Section 4 where appropriate to back up or elucidate an argument. 
In between the two data collection sessions, the participants were in regular daily contact, with the exception of IND, who was a full-time student and could not participate in several of the concurrent group activities due to time constraints. Group 1 took part in events organised at the host institute as part of its regular schedule, including an international workshop and the institute's five-year anniversary. Group 2 participated in a full-time two-week international deaf capacity building programme on the campus of the host university. Both groups also undertook joint trips to other places in the UK and India respectively.

The Group 1 international participants stayed in private accommodation shared with local British deaf people, whereas the Group 2 international participants were accommodated in the gender-separated university hostels. In both cases, there was ample contact with the local deaf communities in Preston and at the university in Lucknow, which had several dozens of deaf students on its residential campus at the time.

\section{Response times in elicitation games}

In this section, the response times by the pairs of signers in the elicitation games are compared between the first round (SET1 data) and the second round (SET 2 data) of the elicitation games. Response times are defined as the time interval (in seconds) between the director starting to sign and the matcher pointing to the picture that they have identified as their response. At each turn, a response was given only once, that is, multiple tries to identify the same target picture were not allowed.

For the game, every signer was paired with every other signer within their group, resulting in six dyads per group. Although a higher error rate, i.e. identification of the wrong target picture, may have been expected in round 1, this was not the case and the vast majority of responses were correct in both rounds of the game.

Table 2 shows the total response times combined by all dyads in SET1 and SET2 in the two groups. As expected, the elicitation game is resolved much faster in SET2, as participants have had several weeks in between in which to adjust their communication to each other. This is shown as "time saved" in Table 2. For Group 1, the second round of the game is $34.66 \%$ faster, and for Group 2, the second round is $51.49 \%$ faster. In Group 2, each pair resolved the task for a total of 20 pictures. 
However, in Group 1, SET2 had more pictures than SET1 in several cases, and the number of SET2 pictures was capped so that both sets had equal numbers. ${ }^{1}$ Despite this slight difference in rigour of the data, the reduction itself is not in question.

\begin{tabular}{ll}
\hline Group 1 total response time SET1 & 2383 \\
\hline Group 1 total response time SET2 & 1557 \\
\hline Group 1 time saved & $826(34.66 \%)$ \\
\hline Group 2 total response time SET1 & 2430 \\
\hline Group 2 total response time SET2 & 1203 \\
\hline Group 2 time saved & $1227(50.49 \%)$
\end{tabular}

Table 2. Total response times

While an overall reduction in the time taken is of course expected, the specific interest here lies in differentiating between the four signers in each group. This is shown in Tables 3-6. The tables show the total response times with respect to the individual participants in SET1, as well as the differences in response times, each time differentiated by the director and the matcher roles in Group 1 and Group 2. In Tables 4 and 6, the total "time saved" between SET1 and SET2 is added up for each participant as director, as matcher, and for both roles combined. These data indicate two related but separate measures: the degree of initial difficulty with the task (SET1 response times), and the degree of headway that signers are making between SET1 and SET2 elicitation ("time saved" data).

It is very clear from the data that the differences in response times vary greatly between participants. For example, the Group 1 response times in pairs where INDO1 was the director is virtually unchanged, while there is a large difference in response times where JP was involved either as director or as matcher. The remainder of Section 3 summarises the patterns found in the data in terms of a) which individual participants stand out as having an unusual profile that differs from

\footnotetext{
${ }^{1}$ The BRT-JP pair was capped at 16 pictures, and JP-JORD1, JORD1-INDO1, and BRT-INDO1 at 21 pictures each, with the remaining pairs having 20 pictures. Response times are based on the capped data. Capping was done sequentially, i.e. taking in all data from the start in succession until the capped number of pictures was reached.
} 
the rest of the group and $b$ ) which pairs of signers show unusual or unexpected patterns that call for an explanation. Possible factors that may be responsible for such differences and particularities are then considered in Section 4, in relation to differences between participants' backgrounds.

\begin{tabular}{lccccc}
\hline Director & SET1 matcher & & & & Totals \\
& JP & JORD1 & INDO1 & BRT & \\
BRT & 246 & 135 & 214 & - & 595 \\
JP & - & 202 & 312 & 182 & 696 \\
JORD1 & 278 & - & 146 & 135 & 559 \\
INDO1 & 288 & 132 & - & 113 & 533 \\
Totals & 812 & 469 & 672 & 430 & \\
\hline
\end{tabular}

Table 3. SET1 response times in Group 1.

\begin{tabular}{lccc}
\hline Time saved & & & \\
& as director & as matcher & in both roles \\
BRT & 270 & 147 & 417 \\
JP & 332 & 368 & 700 \\
JORD1 & 195 & 140 & 335 \\
INDO1 & 29 & 171 & 200 \\
& & & \\
Total & 826 & 826 & \\
\hline
\end{tabular}

Table 4. "Time saved" differentiated by participants, Group 1.

The participant standing out most clearly from amongst the Preston group is JP, the signer from Japan. Dyads involving JP both as director and as matcher have taken the longest time in SET1 by a considerable margin, namely a total of 696 s where JP was acting as director with any of the other participants as matcher, and a total of 812 s where JP had acted as matcher for any of the other signers. This compares to figures of between 533s and 595s total time taken by the other signers acting as director, and between 430 s and 672 s taken by the other signers acting as matchers (Table 3). This pattern points to considerable initial difficulty with the task for JP and those matched with him. Subsequently, these large differences disappear in SET2, where JP is intermediate between the other signers. Consequently, dyads involving 
JP have the highest "time saved" figures, as shown in Table 4 (combined figure of 700s).

The other notable pattern in Group 1 is the fact that INDO1 as director shows almost no difference between the SET1 and the SET2 figures, with only 29s of "time saved". INDO1 also has the lowest total response times as director in SET1, with 533s (Table 3), but its intermediate between the other signers as matcher.

Apparently, INDO1 was a highly successful communicator right from the start in terms of conveying the message. The remaining individuals and pairs in Group 1 do not show any particularities with respect to their response time patterns.

\begin{tabular}{lccccc}
\hline Director & SET1 matcher & & & & Totals \\
& JORD2 & IND & INDO2 & NEP & \\
NEP & 172 & 197 & 162 & - & 531 \\
JORD2 & - & 175 & 161 & 222 & 558 \\
IND & 213 & - & 265 & 344 & 822 \\
INDO2 & 153 & 195 & - & 171 & 519 \\
Totals & 538 & 567 & 588 & 737 & \\
\hline
\end{tabular}

Table 5. SET1 response times in Group 2

\begin{tabular}{lccc}
\hline Time saved & & & \\
& as director & as matcher & in both roles \\
NEP & 252 & 494 & 746 \\
JORD2 & 234 & 282 & 516 \\
IND & 478 & 227 & 705 \\
INDO2 & 263 & 224 & 487 \\
& & & \\
Total & 1227 & 1227 & \\
\hline
\end{tabular}

Table 6. "Time saved” differentiated by participants, Group 2.

Tables 5 and 6 show the equivalent figures from Group 2. In this case, the Indian and the Nepali signer stand out as having the highest "time saved" figures in both roles combined (705s and 746s). This is due to IND having by far the slowest response times in SET1 in the role of director (822s) and NEP having by far the slowest response times as matcher (737s). Moreover, the single highest response time in SET1 data occurs where IND as director is paired with NEP as matcher (344s). Thus despite the geographical proximity between India and Nepal, this pair 
shows no advantage over other pairs in these data. However, additional figures and considerations presented in Sections 4.1 and 4.2 complicate this initial picture.

The remaining individuals and pairs in Group 2 are similar to each other. For the SET1 figures, none of the remaining pairs differs from other pairs by more than $44 \mathrm{~s}$, and the maximum difference between totals is $39 \mathrm{~s}$ for directors and $50 \mathrm{~s}$ for matchers. Similarly, in Table 4 all individual figures other than IND as director and NEP as matcher differ only by a maximum of $58 \mathrm{~s}$, whereas there is a large gap of 196s and 212s respectively between the two high outlier figures and the next highest figure in the data.

In order to try to make sense of such patterns in the data, it is helpful to look at potential factors that may influence the degree of difficulty that signers experience initially, as well as the extent of their journey towards improving their communication with the other signers. This analysis is addressed in Section 4. It is important to note that the error rate, i.e. the number of times that the wrong response was selected by the matcher, cannot be used for the analysis. Errors in the selection of the target picture do not exceed $5 \%$ even in the first rounds, and hardly change between the first and second rounds of the game.

\section{Inhibiting and facilitating factors}

As a first step towards understanding the patterns summarised in Section 3, six factors are highlighted here, each of which could have a positive, negative or neutral effect on the likely communicative success of a given pair of signers. In the case of a positive effect, that is, any factor that could be helpful in improving communication between a pair of signers, the potential factor/effect is called facilitating here. In the opposite case, where a particular factor could pose an obstacle to ease of communication, the potential factor/effect is called inhibiting. Where no particular argument can be made, the factor/effect is called neutral.

It should be recognised from the outset that response times and degrees of communicative ease are extremely complex issues that could be influenced by a large number of factors, some of which cannot be captured in any systematic way. For example, undoubtedly each individual's motivation, attitude, personality and 
culture plays a major role in how the game is perceived and performed: How outgoing and talkative is the person? How seriously do they take the game in terms of getting the responses right? What is the cultural bias in terms of asking for clarifications, or in terms of communicating with the opposite gender? Such factors are likely to influence the way in which the game was approached, despite the initial briefings being the same for everyone. Similarly, the interpretation of response times themselves needs to be approached with caution. Although the response times are used here as a measure of communicative efficiency, it is conceivable that a particular pair may communicate very well but still have a slower response time for reasons unrelated to efficiency, e.g. because they enjoy the interaction, or because they want to make sure that their response is correct. Moreover, the degree of (objective or perceived) difficulty of pictures to be described within the game cannot be fully standardised and may vary unpredictably. The following discussion should be read with these caveats in mind.

Given these complexities, and the interaction between multiple factors, we cannot expect any clear-cut 'proven' conclusions. Moreover, we are dealing with a small number of participants, so that generalisations can only be preliminary. Instead, the aim is to consider the way in which various factors may correlate with the objectively observable data, in order to arrive at a better qualitative understanding of what it means for sign language users to be involved in this kind of communicative situation. Where available and appropriate, other sources of information are also brought to bear on the question. This includes comments made by participants during their introspective interviews, and observations made by members of the research team in the field.

\subsection{Exposure}

The most basic facilitating factor involved in constituting improved communication over time is the amount of opportunity that the participants in each group have to spend time with each other. In Group 1, there were no large differences in this respect, as all participants were generally together as a group during the day at the host institute, and then returned to their separate accommodations overnight. However, in Group 2, participant IND was disadvantaged by not participating in a 
daytime programme along with the others, who were following a full-time leadership development programme for two weeks together with some other international students at the host university. IND was unable to join this programme due to time constraints, as he was a full-time student at the host university at the time. This lack of exposure is visible as an inhibiting factor in the SET2 response times, ${ }^{2}$ where the two longest response times of pairs of signers (266s and 246s) are associated with IND as one of the participants, while all other response times are between 165s and 185s. SET2 figures also reveal that no matter who is the director, the response time in combination with IND is slower than in combination with the other signers, and no matter who is the matcher, the response time is slowest when IND is the director. This is true of all pairs except the IND-NEP pair, which is in line with the other pairs.

These figures suggest that lack of exposure with other cross-signers is an inhibiting factor in general that results in the overall slower response times for IND, in particular in SET2. This picture is complicated by the fact that IND also shows an unusual pattern for the first round of the elicitation game in SET1, as discussed in Section 3. The subsequent figures for the IND-NEP pair in SET2 suggest that the lack of exposure as an inhibiting factor in general may interact with other facilitating factors, such as typological similarity (Section 4.2), specifically for this pair. Thus there is a large degree of improvement in this pair from the initial difficulties to the SET2 figures. In fact, the "time saved" between SET1 and SET2 for the IND-NEP pair is 369s, which is between 140s and 227s more than for the other pairs.

\subsection{Typological distance}

A possible inhibiting factor is when a sign language within a particular group of users of diverse sign languages is typologically more distinctive than the other sign languages. It is not the aim here to measure typological distance in an accurate way, and there is in fact no such methodology. Therefore, the discussion here does not cover all sign languages in the two groups, but is limited to the more straightforward examples. For an overview on the typology of sign languages, see Palfreyman and Zeshan (2017).

\footnotetext{
${ }^{2}$ Response time tables for SET2 are not included in this article as they are not relevant to the majority of factors discussed.
} 
In Group 1, typological distance applies as an inhibiting factor to Japanese Sign Language, which has many particularities not generally shared with other sign languages around the world, including its extensive gender-marking system (see Fischer and Gong 2011), the first-person pronoun pointing to the nose instead of the chest, or differences with respect to metaphorical mappings, communicative gestures and culturally embedded emblems that are common in many other parts of the world. For instance, signs associated with semantics of cognition use the head as place of articulation in most sign languages, but this is not always the case in Japan across the signs in this semantic domain. In the absence of an actual measure, the typological distance of Japanese Sign Language is based on the intuitions and observations made by both participants and members of the research team, and this applies to both lexical and grammatical observations..

In the case of participant JP, the other participants in Group 1 commented on the increased difficulty due to many differences in the Japanese signs. For instance, it was considered surprising that presumably "easy-to-understand" signs such as KNOW (signed at the temple), BABY (movement imitating how a baby is held), HOPE (the emblem with fingers crossed), and LOVER (signed at the heart) were not understood by JP, as the Japanese signs do not use the same metaphorical and iconic mappings as many sign languages in other parts of the world. It makes sense to assume that with exposure over several weeks, these difficulties can be lessened, which is visible in a considerable shortening of response times with JP as participants in the second round of the games. In the SET2 data, JP's response times are intermediate between the other signers both as director and as matcher.

By contrast, sign languages in India and Nepal share a number of structural features, for example the kinship system (both individual signs and the system itself) and some functional particles, which are derived from gestures. Overlaps in some of the kinship signs from India and Nepal are documented in Bradford, Michaelis and Zeshan (forthcoming). A certain level of similarity could be due to geographical proximity and language contact, but the reason for typological similarities does not need to be discussed at this point. Given the figures discussed in Sections 3 and 4.1 with respect to India and Nepal, we may assume that the facilitating effect of typological similarities interacts with other factors that are inhibiting, namely the lack of exposure for IND, thereby producing the complex picture of response times in the IND-NEP pair. 


\subsection{Home advantage}

This factor refers to the fact that in each group, one of the participants (BRT and IND respectively) was in their own country, while the other three had travelled inbound from abroad. Potentially, the participant in their home country has an advantage in communicating because of the strength of their own surrounding sign language (British Sign Language, BSL, in the UK and Indian Sign Language, ISL, in India). As all participants from abroad are exposed to these sign languages in the environment in between the first and second round of elicitation, there is a potential home advantage for the in-country participant. In particular, we might expect that the "time saved" advantage would be greater for the BRT and IND participants because the group as a whole might gravitate towards the surrounding sign language over time, aligning more closely with their own BSL and ISL models. However, the data do not support "home advantage" as a facilitating factor, as BRT and IND do not have larger "time saved" intervals than the other participants.

This conclusion is complicated by findings in Bradford, Michaelis and Zeshan (forthcoming), who report that for the kinship referents investigated, the signers do strongly tend to converge on the forms used in the surrounding environment. By the time of the second elicitation game, Group 2 participants only use the Indian/Nepali signs for MALE and FEMALE (which are identical in both countries), and have dropped all other variants used in the first round of the game. In Group 1, the picture is more complex as two preferred forms for MALE and FEMALE emerge over time, one each from British Sign Language and one each from International Sign. This perhaps reflects the fact that the main language of communication within the UK host institute is International Sign, while British Sign Language is the language of communication outside among the local deaf people. Previous literature on International Sign has reported that the choice of IS lexemes is influenced by the signs used in the surrounding local community and therefore the choice of signs changes according to the local audience (e.g. Mesch 2010).

In spite of these findings, the home advantage factor does not translate into faster response times for BRT and IND, which is somewhat surprising. It may be that in the interplay of various factors, there are other factors that override the potentially facilitating effect of the home advantage factor in both groups. The issue of interplay between factors is discussed further in the conclusion. 


\subsection{Literacy, writing system, and manual alphabet}

In the situation of cross-signing, making use of textual literacy in the form of a manual alphabet (fingerspelling), or writing (in the air or on surfaces, e.g. the palm of the hand) is a semiotic option that signers may use as part of their multimodal repertoires (Zeshan 2015). If participants share a language of literacy, this can potentially be a bridge that facilitates communication. Similarly, sharing the same manual alphabet is potentially a facilitating factor.

In both Group 1 and Group 2, no two participants share the same manual alphabet with respect to their native sign languages. However, the one-handed manual alphabet used in International Sign is a shared resource to some extent, though several participants only have partial, and sometimes marginal, competence, both in the alphabet itself and with respect to shared knowledge of any written/spelled words. Similarly, there is some overlap with respect to writing systems: English and Bahasa Indonesia use the same type of alphabet, while Nepal (for Nepali) and India (for Hindi and other languages) both have a Devanagari-based script, though Indian Sign Language uses a two-handed manual alphabet that is very similar to the alphabet used in British Sign Language and does not represent Hindi or any other indigenous Indian language. Recourse to fingerspelling and/or writing is usually with respect to English in the data, and writing is used for expressing numerals too. Zeshan (2015) details how the use of writing in cross-signing is influenced by the literacy practices of the signers' home countries.

When cross-checking these shared literacy-based resources with response times from the data, no positive correlation is found. That is, none of the pairs of signers who share the same writing system shows any faster response times compared with those pairs where both individuals have different writing systems. Thus literacy-based shared resources do not have any evident facilitating effect on how well the signers communicate in the elicitation experiments. This is in line with the findings in Byun et al (forthcoming), who investigate repair strategies (i.e. how signers resolve misunderstandings) in the casual conversations of cross-signers. These findings show that literacy-based strategies result in successful repair less than half of the time, and among all the options investigated, this is one of the least successful strategies. Nevertheless, literacy is sometimes used as a bridge in cross- 
signing communication, as in this example that was noted in one of the interview transcripts (see also Byun et al, forthcoming, for other examples):

He has written something in English letters on the palm of his hand. He is trying to process what the UK partner has fingerspelled and he believes that writing the letters on his palm might help.

For the opposite effect, that is the lack of shared literacy resources as an inhibiting factor, we do not have clear evidence either, though it is still possible that some signers have experienced this as more of a barrier than others. For instance, notably the Japanese and the Jordanian participants have writing systems that are more divergent, with a syllabic script for Japanese and a right-to-left script without short vowels in Arabic. However, it is not clear from the data whether this has posed additional difficulties.

\subsection{Variability in visual-gestural communication environments}

This factor refers to the breadth of variation in the visual-gestural communication that each participant has experienced in their home area. This includes both the native sign language environment, and visual-gestural communication with hearing people.

With respect to the participants' native sign languages, their level of dialectal diversity varies tremendously across locations. For instance, Japanese Sign Language in JP's home area of Tokyo is comparatively homogenous; the Tokyo dialect is considered a standard variety across most of Japan, and dialectal diversity has been reducing in Japan (Sagara 2014). At the other end of the spectrum, the sign language situation in Indonesia is highly multi-dialectal (Palfreyman, forthcoming). For instance, INDO1's home city has five different numeral paradigms that all co-exist with each other. In fact, Palfreyman 2014:243 observes that in Indonesia "there is more variation within urban centres than between them". Both INDO1, from Solo in Java, and INDO2, from Makassar in South Sulawesi, are highly multi-dialectal due to exposure within their home cities and contact with signers from across Indonesia.

In terms of communicative resources to be deployed in cross-signing, signers from areas with a greater extent of dialectal variation could be at an advantage because they have more experience with lexical flexibility and with communicative strategies to resolve the meaning of unfamiliar signs. Indeed, we see that INDO1 and INDO2 are particularly successful as directors in the first rounds of the game, 
with the fastest combined response times in SET1 (shown in the rightmost column in Tables 3 and 5). This advantage does not seem to extend to the role of matcher. However, they both have low figures with respect to the "time saved" in both roles combined, particularly evident for INDO1, and this may indicate that overall, their journey over time has not had to be as pronounced as for some of the other signers in terms of overcoming initial difficulty.

Secondly, this factor also takes into account the extent to which participants have had experiences of gestural communication with hearing people. From the conversational data and the introspective interviews conducted with the participants, we know that in the cross-signing situation, they draw on these experiences and strategies. For example, the interview transcript with JORD1 states:

The Jordanian signer uses the two-finger sign "same-same", not the other Jordanian sign. He said he changed it because that is the gesture hearing people typically use in Jordan, and he thought it would be more readily understood.

Another example is JP's use of two-handed numeral signs that are not part of Japanese Sign Language but are sometimes used as gestures in Japan (Zeshan 2015, Sagara 2014).

These findings support the hypothesis expressed in Crasborn and Hiddinga (2015:66) that "deaf-hearing encounters feature the exact same communication strategies that deaf people use in first-time encounters with deaf people from other sign language backgrounds".

With respect to the sign language environment at home, it is notable that three of the participants, NEP, INDO1, and JP, grew up in a family with deaf parents, siblings, and/or other close deaf relatives. In view of earlier research, we may have expected this to be a facilitating factor, given that signers from deaf families have been found to have higher sign language skills than those from hearing families, due to the fact that deaf families facilitate language acquisition from the earliest stages of life (Boudrault and Mayberry 2006; Mayberry and Eichen 1991). However, the data show no such advantage for this sub-group of participants.

For a potential explanation, one consideration is that growing up in a signing environment with a network of deaf family and friends results in fewer instances of having to negotiate communication in deaf-hearing encounters, at least during childhood. A reduced amount of deaf-hearing interactions may be a disadvantage with respect to cross-signing, although this point would need to be substantiated with 
suitable participant background data (e.g. interviews about both childhood and adult life).

\section{Conclusions}

This article has explored a range of facilitative and inhibiting factors that affect how adeptly cross-signers can meet the challenges of cross-signing, and has put these into correlation with a quantitative measure that reflects communicative ease, namely the response times for an elicitation game. In these data, it appeared that exposure to the other group members, typological distance, the family constellation in terms of growing up with deaf family members, and the dialectal diversity all may have influenced the level of communicative ease among participants. On the other hand, no evidence was found for the surrounding sign language (home advantage) or shared literacy resources being impactful factors, and they appeared to be neutral at this stage of the research.

I also argued that the factors described in Section 4 cannot be said to be proven or disproven in any quantitatively robust sense because there are too many tightly interwoven aspects to this type of communicative situation, and the number of participants is low. Instead, this article has made a first-pass attempt at a better understanding of the role that multiple interrelated factors may play in situations of cross-signing. In doing so, finding a lack of any correlation between hypothesised factors and quantifiable data has at times been just as revealing as finding evidence for likely inhibiting or facilitating effects. In future research, similar studies could be conducted with larger numbers of participants in order to corroborate, discard, or add to the factors proposed here. In doing so, care must be taken to keep a holistic view on cross-signing situations and their complexities rather than trying to study individual factors in isolation.

Within previous research on IS, the main focus has been on the output produced by signers rather than on comprehension, with few exceptions such as Whynot (2015). Cross-signing research has used specific methodologies, including introspective interviews, to address the issue of comprehension (Zeshan and Webster, forthcoming). Such methodologies allow us to develop a more specific and differentiated view of what it means to say that deaf sign language users are able to communicate across language boundaries with relative ease. Further studies on 
comprehension of both cross-signing and semi-conventionalised IS would be very valuable.

Two further tentative conclusions can be drawn from the discussions in this article. Firstly, inhibiting and facilitating effects may be cumulative. That is, the effect, here measured by proxy in terms of response times in the elicitation games, will be strongest if several factors are acting together in favour or against the potential for smooth communication in the early stages of cross-signing. For example, participant JP had to contend with a number of potentially inhibiting factors: the typological distance between Japanese Sign Language and the sign languages of the other participants; the low level of dialectal variation in Tokyo; and potential influence from divergent writing and fingerspelling systems.

Secondly, some of the factors seem to have much stronger effects than others. Unsurprisingly, this applies to lack of exposure (Section 4.1), as crosssigning communication develops as a result of face-to-face contact. Thus IND has been disadvantaged by this factor, overriding other potentially facilitating factors such as his experience of studying in a group of international deaf students for four years. As far as facilitating factors are concerned, the sum total of a person's experience of overcoming communication difficulties, be it with hearing non-signers or with users of other sign language dialects, seems to be particularly important. The inter-relationship between various factors is likely to be of particular interest for future research.

References:

Bauman, H-Dirksen \& Joseph J. Murray. 2014. Deaf gain. Minneapolis: University of Minnesota Press.

Bauman, H-Dirksen \& Joseph J. Murray. 2009. Reframing: From hearing loss to deaf gain. Deaf Studies Digital Journal 1. 1-10.

Boudreault, Patrick \& Rachel I. Mayberry. 2006. Grammatical processing in American Sign Language: Age of first-language acquisition effects in relation to syntactic structure. Language and Cognitive Processes 21(5). 608-635. 
Bradford, Anastasia, Susanne Michaelis \& Ulrike Zeshan. Forthcoming. Stabilisation of the lexicon in an emerging jargon: The development of signs to express animate referents in a sign language contact situation. In Ulrike Zeshan \& Jennifer M. B. Webster (eds), Sign Multilingualism.

Byun, Kang-Suk, Connie De Vos, Ulrike Zeshan \& Stephen Levinson. Forthcoming Communicative success of repair strategies in cross-signing. In Ulrike Zeshan \& Jennifer M. B. Webster (eds.), Sign Multilingualism.

Fischer, Susan D. \& Qun-hu Gong. 2011. Variation in East Asian sign language structures. In Diane Brentari (ed.), Sign Languages, 499-518. Cambridge: Cambridge University Press.

Friedner, Michele \& Annelies Kusters (eds.). 2015. It's a small world: International deaf spaces and encounters. Washington, DC: Gallaudet University Press.

Gass, Susan M. \& Alison Mackey. 2000. Stimulated recall methodology in second language research. New York \& London: Routledge.

Green, E. Mara. 2014. Building the tower of Babel: International Sign, linguistic commensuration, and moral orientation. Language in Society 43(4). 445-465.

Hansen, Martje. 2015. What Is International Sign? The Linguistic Status of a Visual Transborder Communication Mode. In Rosenstock, Rachel \& Jemina Napier (eds), International Sign: Linguistic, usage, and status issues, 15-32. Washington, DC: Gallaudet University Press.

Hiddinga, Anja \& Onno Crasborn. 2011. Signed languages and globalization. Language in Society 40(4). 483-505.

Hockett, Charles F. 1960. The origin of speech. Scientific American 203:88 96.

Kusters, Annelies \& Michele Friedner. 2015. Deaf-same and Difference in International Deaf Spaces and Encounters. In Michele Friedner \& Annelies Kusters (eds.), It's a small world: International deaf spaces and encounters, ix-xxix. Washington, DC: Gallaudet University Press.

Mayberry, Rachel I. \& Ellen Eichen. 1991. The long-lasting advantage of learning sign language in childhood: Another look at the critical period for language acquisition. Journal of Memory and Language 30. 486-512.

McKee, Rachel \& Jemina Napier. 2002. Interpreting in International Sign Pidgin: An analysis. Journal of Sign Language Linguistics 5(1). 27-54.

Mesch, Joanna. 2010. Perspectives on the concept and definition of International Sign. Helsinki: World Federation of the Deaf.

Murray, Joseph. 2008. Co-equality and transnational studies: Understanding Deaf lives. In H-Dirksen Bauman (ed.), Open your eyes: Deaf Studies talking, 100-110. Minneapolis: University of Minnesota Press. 
Palfreyman, Nick. Forthcoming. Variation in Indonesian Sign Language (Sign Language Typology Series No. 7). Lancaster: Ishara Press \& Berlin: De Gruyter Mouton.

Palfreyman, Nick. 2014. Sign language varieties of Indonesia: A linguistic and sociolinguistic investigation. Preston: University of Central Lancashire PhD dissertation.

Palfreyman, Nick \& Ulrike Zeshan. 2017. Sign language typology. In Alexandra Y. Aikhenvald \& Robert M.W. Dixon (eds), 178-216. The Cambridge Handbook of Linguistic Typology, Cambridge: CUP.

Panda, Sibaji \& Ulrike Zeshan. 2015. Two languages at hand: Code-switching in bilingual deaf signers. Sign Language \& Linguistics 18(1). 90-131.

Rosenstock, Rachel. 2008. The role of iconicity in International Sign Language. Sign Language Studies 8(2).131-159.

Rosenstock, Rachel \& Jemina Napier (eds). 2015. International Sign: Linguistic, usage, and status issues. Washington, DC: Gallaudet University Press.

Sagara, Keiko. 2014. The numeral system of Japanese Sign Language from a crosslinguistic perspective. Preston: University of Central Lancashire MPhil dissertation.

Zeshan, Ulrike. 2015. "Making meaning": Communication between sign language users without a shared language. Cognitive Linguistics 26(2). 211-260.

Zeshan, Ulrike, Keiko Sagara \& Anastasia Bradford. 2013. Multilingual and multimodal aspects of "cross-signing" - A study of emerging communication in the domain of numerals. Poster presented at the Theoretical Issues in Sign Language Research (TISLR11) conference, University College London, 10-13 July.

Zeshan, Ulrike \& Jennifer M. B. Webster (eds.). Forthcoming. Sign Multilingualism (Sign Language Typology Series No. 8). Lancaster: Ishara Press \& Berlin: De Gruyter Mouton.

Acknowledgements:

The research leading to these results has received funding from the European Research Council under the European Union's Seventh Framework Programme; we are grateful for funding of this research under the project "Multilingual behaviours in sign language users" (MULTISIGN), Grant Agreement number 263647.

I am very grateful to the participants in the study: Claire Perdomo, Masaomi Hayashi, Muhammad Isnaini Nur Hidayat, Mohammad Abd Elhakim Mohammad Salha, Baha' Mustafa Mohammad Freihat, Muhammad Adam Malik, Navneet Gupta, and Anita Sharma. Other members of the iSLanDS Institute who were members of the wider research team are also gratefully acknowledged: Paul Scott, Nick Palfreyman, Keiko Sagara, and Anju Gurung, who supported the research process as facilitators in many crucial ways; Sibaji Panda who coordinated the research in India; Jennifer 
Webster who scored and cross-checked some of the data; Sam Lutalo-Kiingi and Anastasia Bradford who implemented parts of data collection and participant briefings. I am grateful to two anonymous reviewers and to the volume editor for comments on an earlier version of this article. 